\title{
Chest Ultrasonography in the ICU
}

\author{
Greta Gardelli MD, Francesco Feletti MD, Andrea Nanni MD, Maurizio Mughetti MD, \\ Antonella Piraccini MD, and Maurizio Zompatori MD
}

\author{
Introduction \\ Equipment and Examination Techniques \\ Normal Findings \\ Pneumothorax \\ Pleural Effusion \\ Parenchymal Consolidation \\ Alveolar-Interstitial Pathology \\ Differential Diagnosis in Respiratory Insufficiency \\ Recruitment \\ The Role of Ultrasound in Central Venous Catheter Placement and \\ Arterial Catheterization \\ Limitations \\ Summary
}

Chest diagnostic imaging is essential when dealing with a critically ill patient. At present, direct visualization of the lung parenchyma is performed with a chest $x$-ray and computed tomography with the patient in the supine position. The relative ease of bedside ultrasound examination and the availability of user-friendly, inexpensive, portable equipment have made chest ultrasonography an interesting and alternative method in various situations, because it offers accurate information that is of therapeutic and diagnostic relevance. We describe equipment and examination technique, normal findings, and chest ultrasonography signs detected in some pathological situations, such as pneumothorax, consolidations, pleural effusions, ARDS, and pulmonary edema. Key words: ultrasonography; chest; intensive care. [Respir Care 2012;57(5):773-781. (C) 2012 Daedalus Enterprises]

\section{Introduction}

Chest diagnostic imaging is essential when dealing with a critically ill patient. At present, direct visualization of the lung parenchyma is performed with a chest $\mathrm{x}$-ray (CXR)

\footnotetext{
Drs Gardelli and Mughetti are affiliated with the Unità Operativa di Radiologia; and Dr Nanni is affiliated with the Unità Operativa di Anestesia Rianimazione, Ospedale M Bufalini, Cesena, Italy. Drs Feletti and Piraccini are affiliated with the Unità Operativa di Radiologia, Presidio Ospedaliero di Ravenna, Ravenna, Italy. Dr Zompatori is affiliated with the Dipartimento Immagini, Ospedale S Orsola Malpighi, Università di Bologna, Bologna, Italy.
}

The authors have disclosed no conflicts of interest. and computed tomography (CT), with the patient in the supine position. In the ICU, CXRs are only performed on patients in the supine position. The X-ray beam is positioned directly onto the chest at a distance that is less than satisfactory. In fact, when the x-ray beam does not focus tangentially on the diaphragm dome and the mediastinal structures, a correct diagnosis of the "silhouette sign" may

\footnotetext{
Correspondence: Francesco Feletti MD, Unità Operativa di Radiologia, Presidio Ospedaliero di Ravenna, Ospedale Santa Maria Delle Croci, 5 Viale Vincenzo Randi, Ravenna 48121 Italy. E-mail: francesco.feletti@libero.it.
}

DOI: $10.4187 /$ respcare. 01743 
not be achieved. These circumstances may result in diagnostic errors regarding pleural effusions, parenchymal consolidation, and alveolar-interstitial syndrome. ${ }^{1}$

CT scan assures accurate diagnosis of pneumothorax (PNX), pleural effusion, lung consolidation, and atelectasis. It also serves as a useful guide for chest drainage in localized PNX, abscess, and emphysema. However, this procedure requires that the patient be transported to a $\mathrm{CT}$ unit, which can be risky and requires cardio-respiratory monitoring as well as medical assistance. The risk of overexposure to ionizing radiation is not to be underestimated when repeating examinations to further investigate and follow up a pathological condition.

The relative ease of bedside ultrasound examination and the availability of user-friendly, inexpensive, portable equipment have made chest ultrasonography (CUS) an interesting alternative method in different situations, because it offers accurate information that is of therapeutic and diagnostic relevance. This is why it is often used by intensivists who ask radiologists for a second opinion on difficult or complicated case studies. Experienced radiologists can integrate ultrasound findings with radiological techniques, which means that the contribution of a radiologist is often necessary.

\section{Equipment and Examination Techniques}

CUS is performed using a 3-5 MHz convex transducer, which can visualize deeper lung structures. A high-frequency $5-12 \mathrm{MHz}$ linear array probe is most effective in visualizing the chest wall, pleura, and the lung peripheral parenchyma. A complete examination of the chest requires longitudinal, transversal, and oblique-array probes to be placed along the rib spaces, proceeding from top to bottom in the ventral-dorsal direction, along parasternal, medial clavicular, anterior axillary, medial, and posterior lines. When it is possible to mobilize the patient, probes should also be placed along interscapular and paravertebral lines. The upper clavicular approach assures examination of the brachial plexus and lung apices, while the anterior mediastinal structures can be assessed through the upper sternum. Positioning the probe under the xiphoid with cranial inclination allows evaluation of the pericardium. Subcostal and intercostal acoustic windows of the liver and the spleen are used to study the lung base and pleural effusion.

\section{Normal Findings}

Normally, sectioned images of the ribs in the context of the soft tissue of the chest wall, obtained using longitudinal probes, reveal evident posterior acoustic shadows. In the underlying pleura line, which is clearly hyperechogenic, pleural sliding, called lung sliding or gliding sign, can be detected during breathing. The ultrasound does not

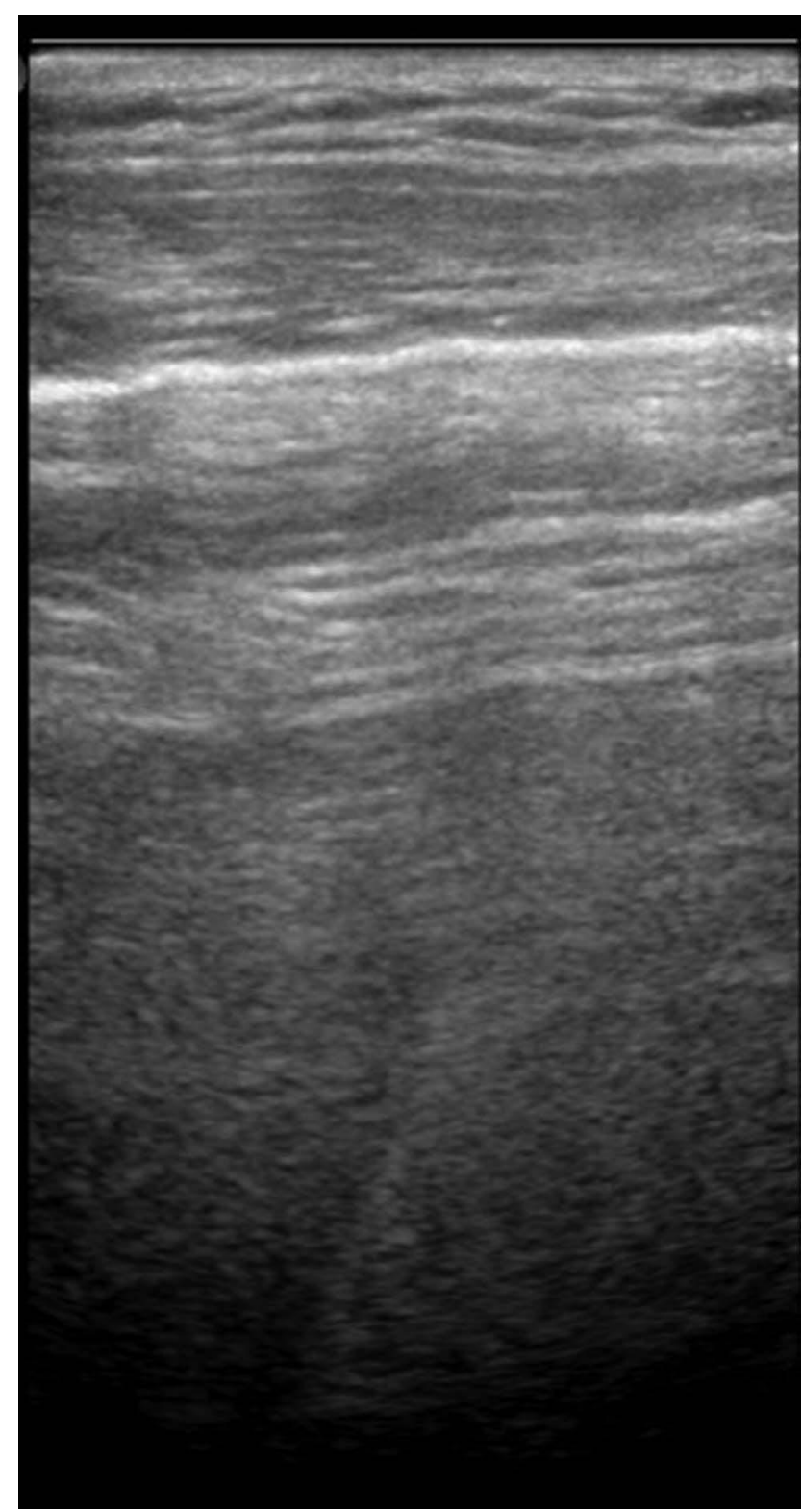

Fig. 1. "A lines": artifacts reproducing the pleural line at regular intervals; this is the time required to return the ultrasound beam to the transducer after being reflected one or more times by the pleural line.

cross the underlying air-filled anatomical structures, and normal parenchyma is not visible. The air creates a reflection of the ultrasound, which is manifested by typical reverberation artifacts: linear images, parallel to the pleural line, which becomes less intense with depth; these are called "A lines" (Fig. 1).

\section{Pneumothorax}

Compared to CXR performed on a supine patient, CUS proves to be more sensitive in detecting PNX. ${ }^{2}$ Lung slid- 


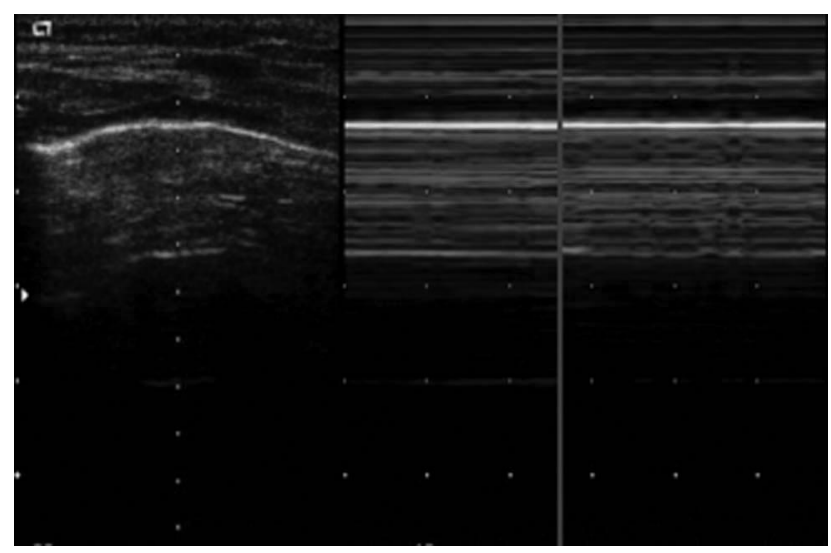

Fig. 2. Pneumothorax characteristic ultrasound appearance with M-mode: barcode.

ing confirms contact of the pleural surfaces and the presence of correct dynamic breathing; this excludes PNX with a $100 \%$ negative predictive value. ${ }^{3}$ Lung sliding is abolished in PNX, and if it is partial, the lung sliding is interrupted at the point where pleural tissue disconnects; this point is well defined in ultrasound imaging and is known as the "lung point." If PNX is suspected on the basis of CUS findings, further confirmation can be obtained by the use of the "M-mode," which highlights a characteristic barcode aspect (Fig. 2).

Lung point is a specific sign of $\mathrm{PNX},{ }^{4}$ but it is not sensitive ${ }^{5}$ because it cannot be detected in massive PNX. Through the visualization and mapping of lung points that can be identified on the chest wall, the CUS allows the extent of PNX to be defined and can indicate the appropriate therapeutic approach. Lung points can be seen along the anterior parasternal line when small areas of anterior PNX are present; these are often not visible with the CXR. On the contrary, identification of these areas at the axillary level indicates larger PNX areas. The examination for PNX should focus on the nondependent areas where it tends to develop, but if the patient is in the supine position, the examination should focus on the anterior parasternal line.

\section{Pleural Effusion}

Ultrasound is more efficient than a normal physical examination $^{6}$ and more useful than CXR in pleural effusion diagnosis. ${ }^{7}$ Even minimal pleural effusion (about $5 \mathrm{~mL}$ ), which is not evident on a CXR, can be detected. If the CXR demonstrates a hemithorax opacity, the CUS can detect quickly and with certainty the nature of the opacity, differentiating liquid and solid components of the opacity (Fig. 3). Transudates are normally anechoic, while exudates are corpuscular (Fig. 4). Hyper-reflectant air bubbles in effusion suggest hydropneumothorax.

CUS is the most accurate technique in indicating the organized or septate nature of effusion (Fig. 5) and can help to avoid the ineffectiveness of evacuative thoracentesis. It is more sensitive and specific than a CT in indicating the presence of septae in pleural effusion. In both cases, CT with contrast injection can reveal a homogeneous hypo-dense aspect, independent of the presence or absence of septations in the pleural effusion. Statistically, CUS is also more accurate in quantifying pleural effusion than a CXR. ${ }^{8}$ The distance between the posterior chest wall and the posterior lung margin represents the total

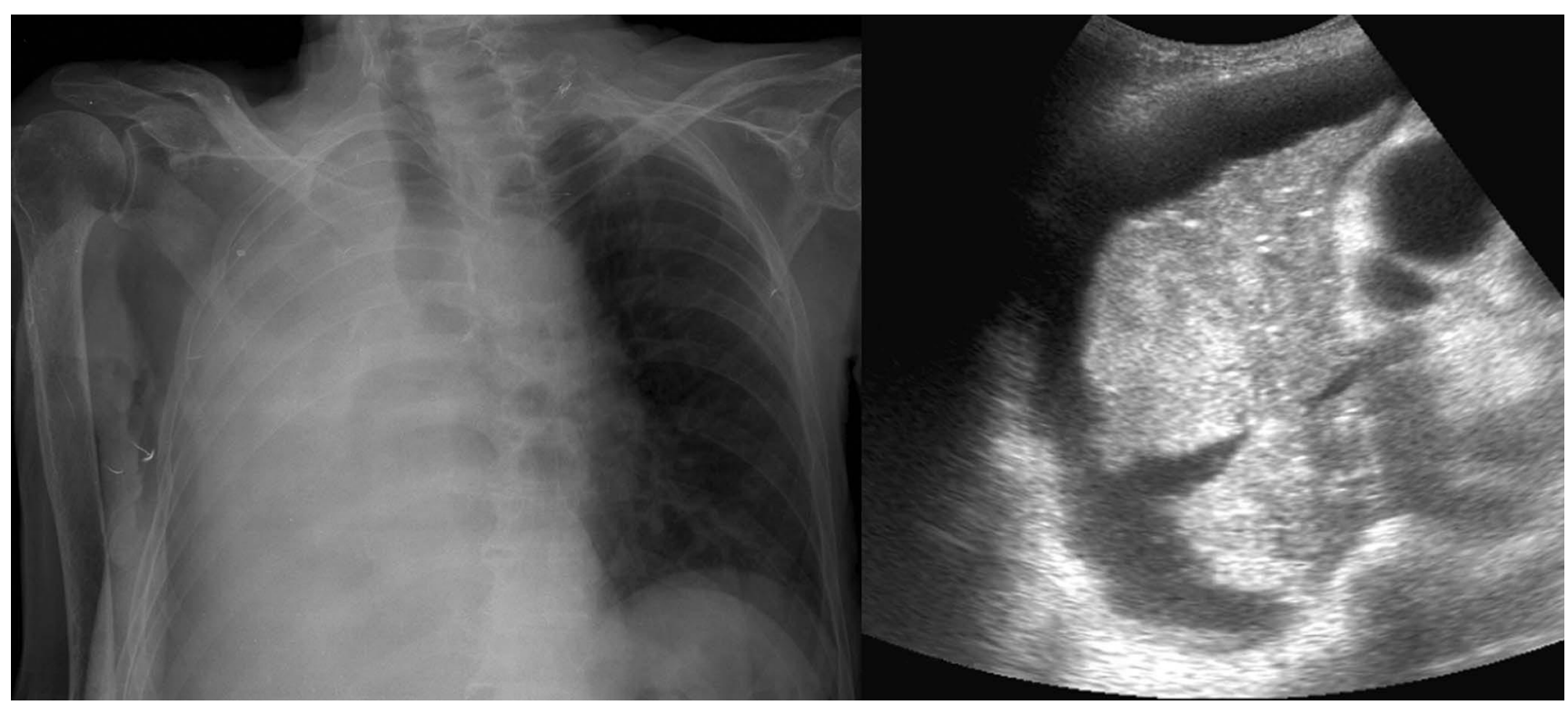

Fig. 3. Opaque hemithorax: chest ultrasonography shows pleural effusion and a large parenchymal consolidation. 


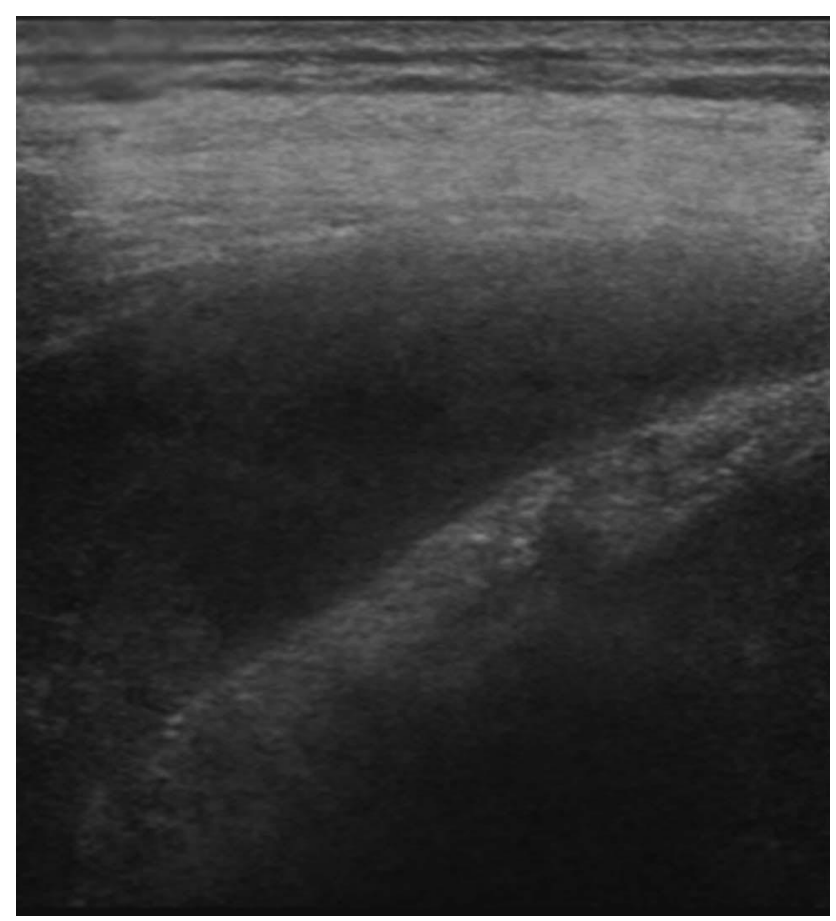

Fig. 4. Corpusculated exudate.

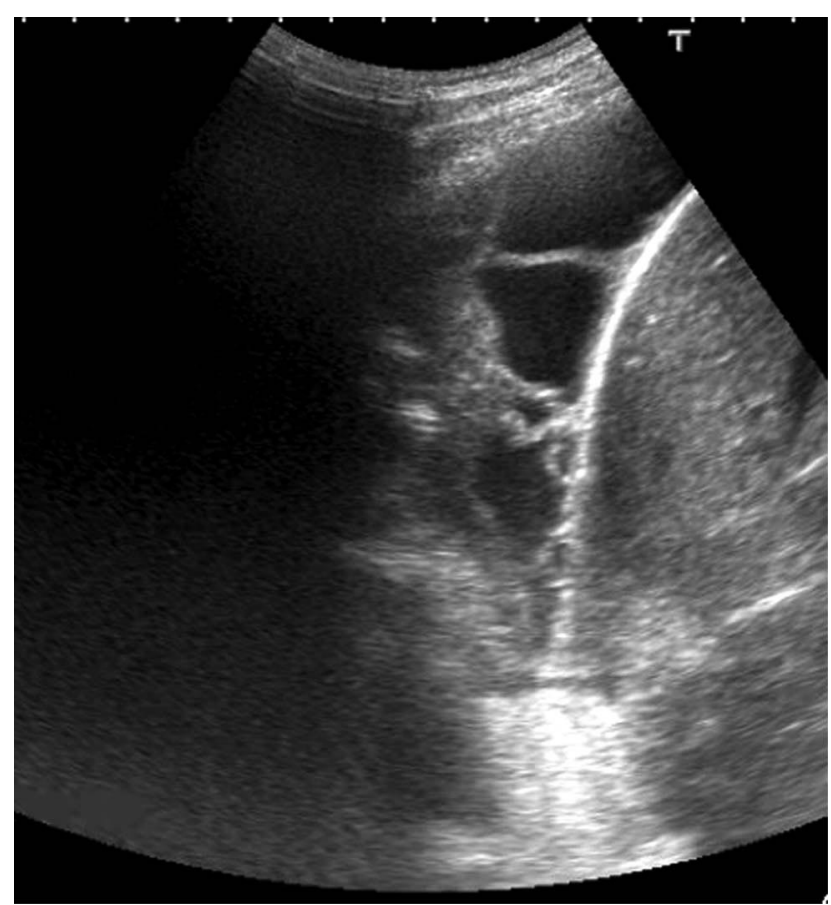

Fig. 5. Pleural effusion with septae.

volume of the effusion. ${ }^{9}$ Roch et al $^{10}$ suggested another technique, by using the distance between the posterior chest wall and the lung at the $5^{\circ}$ intercostal space, $3 \mathrm{~cm}$ above the lung base. If this distance is more than $5 \mathrm{~cm}$, the effusion will be more than $500 \mathrm{~mL}$.

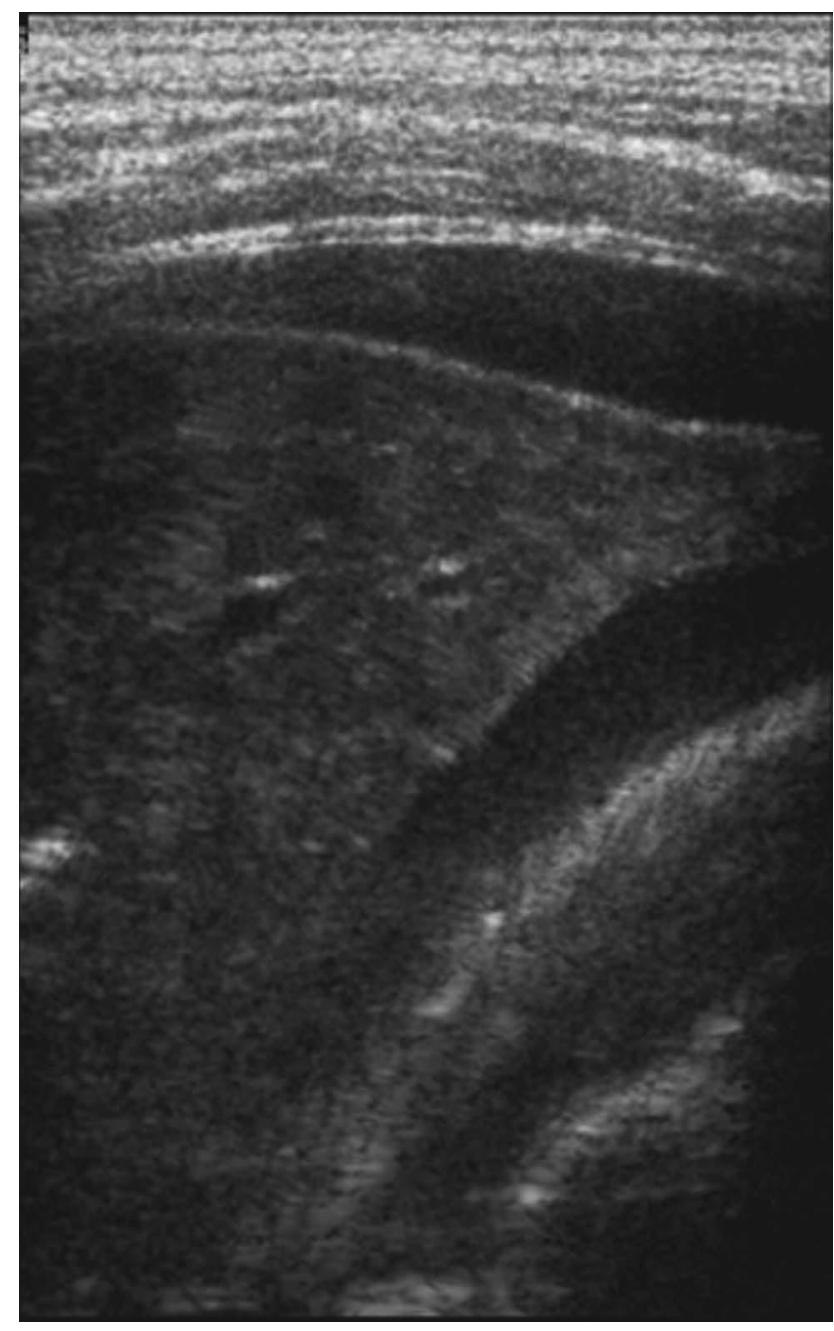

Fig. 6. Pulmonary hepatization in lobar pneumonia.

\section{Parenchymal Consolidation}

The loss of alveolar physiological content allows examination of the lung parenchyma using ultrasound, both when the area of interest is next to the pleura and through the acoustic window opened by pleural effusion. This situation is seen in conditions such as atelectasis or when the alveolar space is filled with exudates and cellular debris, or when the lung parenchyma is destroyed by the proliferation of neoplastic tissue. The consolidations are hypoechogenic, more or less homogeneous, and on CUS examination appear similar to liver parenchyma (Fig. 6).

Consolidations of an inflammatory nature have an irregular profile, with a hypo-anechoic heterogeneous structure and a branching bronchogram. When present, the air bronchograms resemble reverberant ribbon images with a branch-shaped aspect. Their sonographic appearance resembles the hyper-reflective images of biliary tract during aereobilia (Fig. 7), demonstrating a similar branching as- 


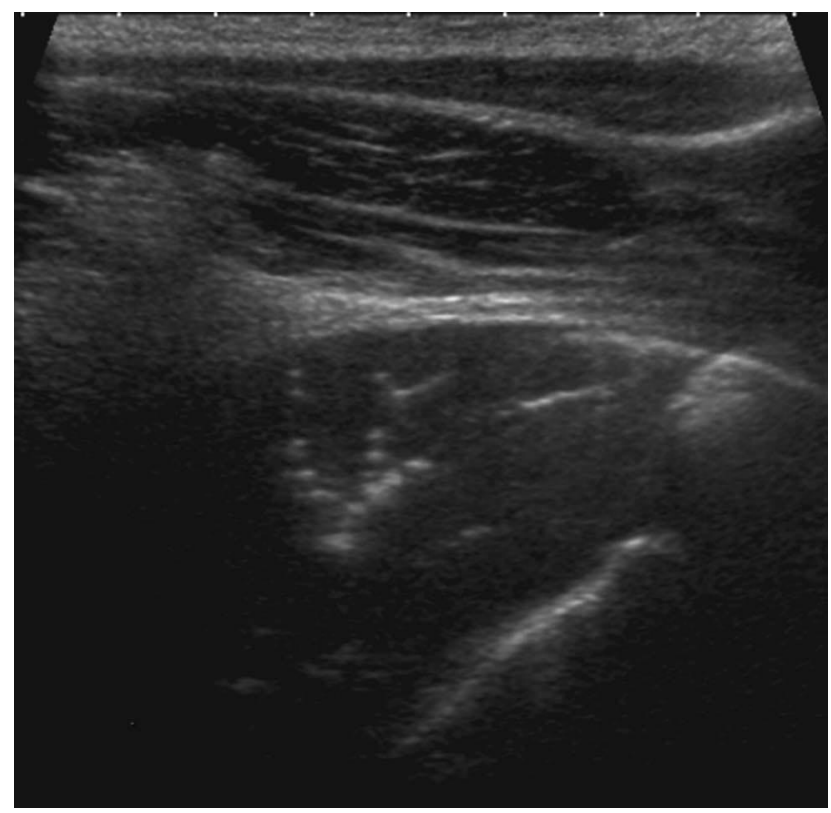

Fig. 7. Air bronchogram.

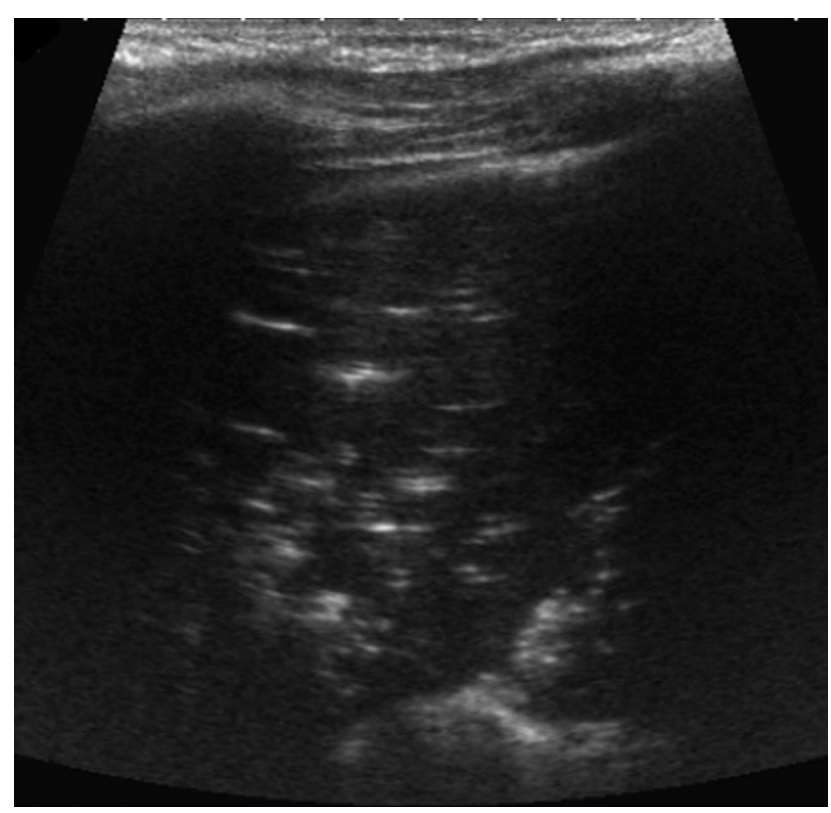

Fig. 8. Atelectasis: horizontal air bronchogram.

pect. Due to loss of volume during atelectasis, the bronchial tubes present a parallel aspect, rather than a branching one (Fig. 8), the latter of which is typical of flogistic consolidations.

In bronchial obstruction, a fluid bronchogram may be observed. The bronchus resembles tubular images, with hyperechoic walls and anechoic content. Figure 9 shows an algorithm used for the differential diagnosis of pulmonary consolidation, based on the aspect of the bronchogram.

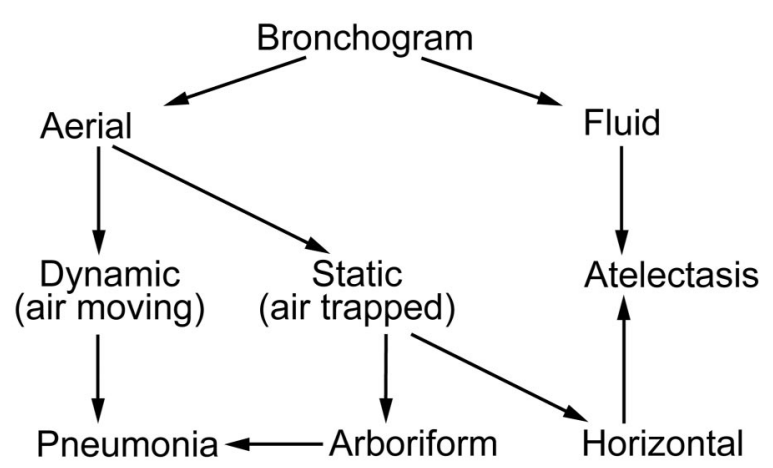

Fig. 9. Algorithm used for the differential diagnosis of pulmonary consolidation, based on the aspect of the bronchogram

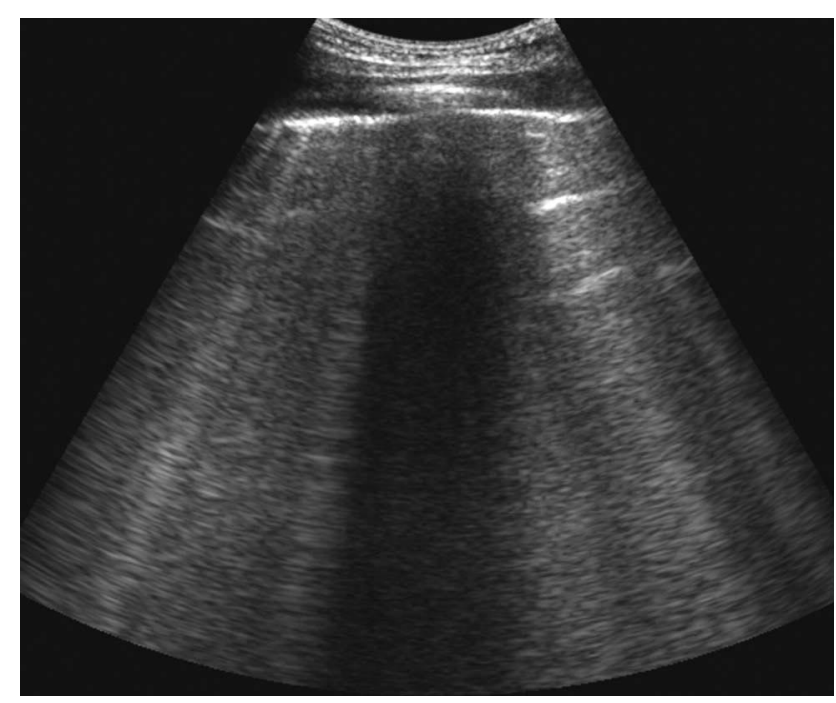

Fig. 10. "B lines."

CUS is a promising technique as a means of diagnosis and can be employed in the monitoring of consolidative pathology until its complete resolution, which avoids the use of $\mathrm{x}$-rays.

\section{Alveolar-Interstitial Pathology}

Under normal and certain pathological conditions (PNX and emphysema), the parenchymatous lung may present a reverberation phenomenon known as "A lines". During pathological processes, which determine the thickening of peripheral interlobular septae, hyperechoic artifacts (ringdown artifacts) appear perpendicular to the pleural line from which they penetrate in depth. These separate slowly and present a radial disposition. These ring-down artifacts are called "B lines" (Fig. 10). Their number reflects the extent of pulmonary edema. When 3 or more "B lines" are seen, interstitial edema can be diagnosed with $97 \%$ sensitivity and $95 \%$ specificity. ${ }^{11}$ 


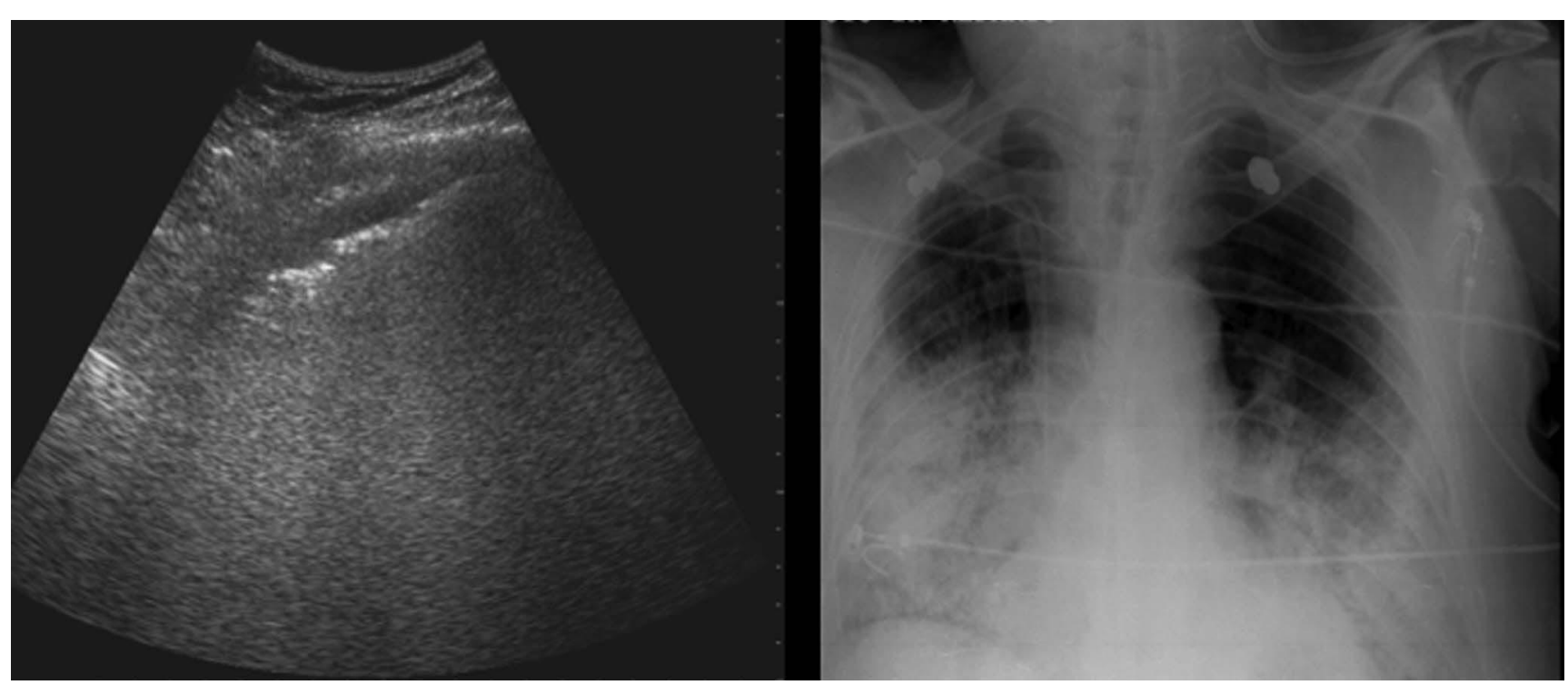

Fig. 11. Pulmonary edema. Radiographic and chest ultrasonography aspect.

As the edema progresses, the "B lines" number increases, they become confluent, and the lung presents a diffuse reflective aspect known as "white lung." A few "B lines" can be also seen in asymptomatic patients and patients without respiratory pathologies, particularly in elderly patients. CUS can detect an increase in the number of "B lines" in elderly but otherwise healthy patients. This is comparable to the situation observed for interstitial pathology signs frequently diagnosed with CT scan in these subjects, as reported by Copley et al. ${ }^{12}$

These findings are not present in young healthy adults. The presence of "B lines" in asymptomatic subjects may reflect physiological modifications of the lung, occurring with increasing age, which are more evident in subpleural areas (eg, thickening of the interlobular septa).

\section{Differential Diagnosis in Respiratory Insufficiency}

Certain authors propose CUS as a valuable instrument in diagnosing the causes of respiratory insufficiency; its use in the ICU has already been described, ${ }^{11}$ and good correspondence was found between the initially identified ultrasound signs and the final diagnosis, with an accuracy of over $90 \%$. Other authors ${ }^{13}$ suggest the use of CUS to differentiate lung cardiogenic edema (Fig. 11) from ARDS (Fig. 12), using the ultrasound characteristics presented in Table 1.

\section{Recruitment}

When treating patients affected by ARDS with mechanical ventilation and PEEP, it is necessary to assess the efficacy of the recruitment maneuvers. The results can be measured in different ways. Currently, the methods of reference are blood gas analysis and CT scan. To date, the only technique employed in assessing the efficacy of recruitment maneuvers in the consolidated parenchyma areas is the CT scan performed before and after recruitment maneuvers. ${ }^{14}$ This, however, involves transporting a critically ill patient, and the results are limited by a static approach.

Recently we suggested CUS for this purpose, with promising results. ${ }^{15}$ The evaluation of recruitment maneuver efficacy by means of CUS can be carried out at the patient's bedside. The advantages of this approach include the reduction of risks connected to the transportation of a patient, as well as the ability to gradually increase ventilation pressure and reduce the risk of barotrauma without exposing the patient to radiation.

In our experience, the recruitment maneuvers can be considered effective if at least one of the following elements is present:

- Reduction $(>1 \mathrm{~cm})$ of the maximum diameter, or reduction of the consistency and homogeneity of the largest consolidation (direct application during the examination)

- Reduction $(>1 \mathrm{~cm})$ of the maximum diameter of at least one of the other consolidations

- Reduction of the identifiable consolidations after the maneuver

The recruited areas become hyper-reflective, similar to the normal lung, but with many "B lines," due to interstitial congestion (Fig. 13). When evaluating blood gas analysis, an increase in $\mathrm{P}_{\mathrm{aO}_{2}} / \mathrm{F}_{\mathrm{IO}_{2}}$ by $>20 \mathrm{~mm} \mathrm{Hg}$ and a $\mathrm{P}_{\mathrm{aCO}}$ 


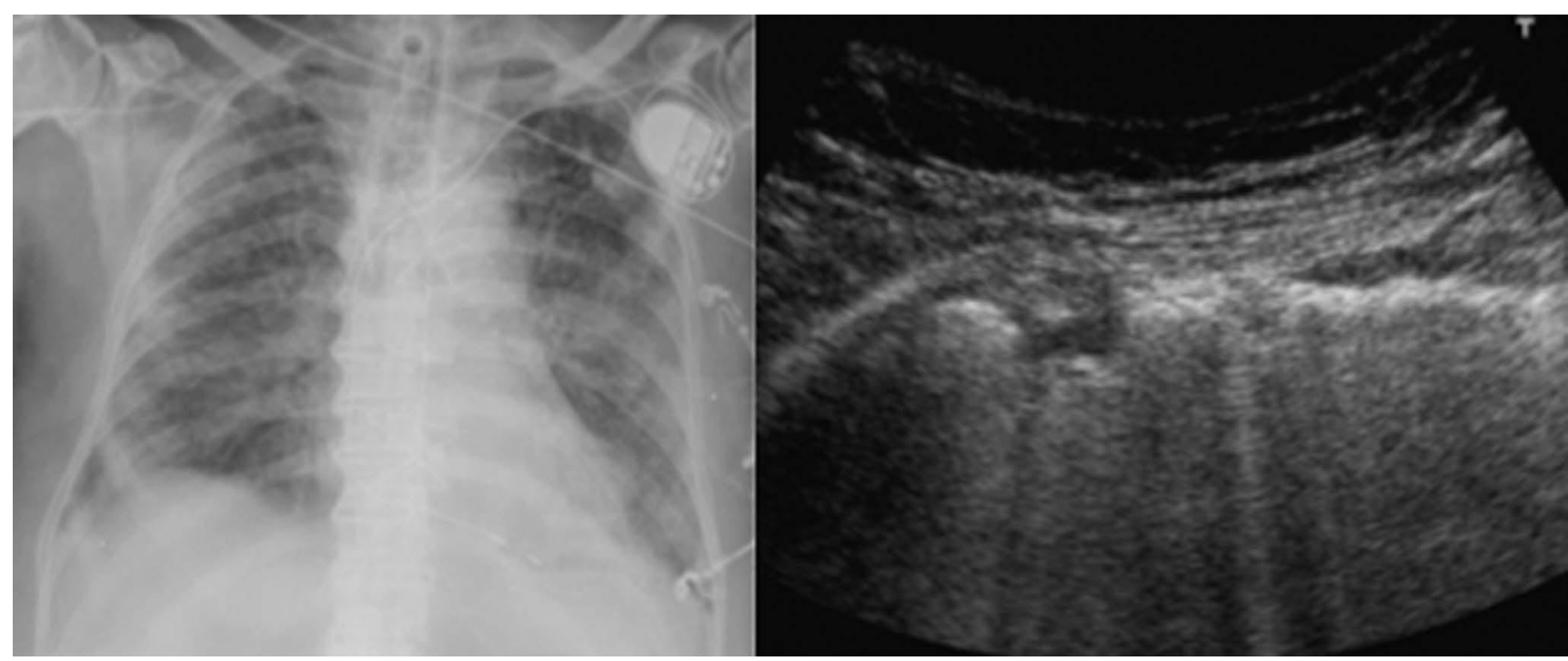

Fig. 12. ARDS. Radiographic and chest ultrasonography aspect.

Table 1. Use of Chest Ultrasonography to Differentiate Lung Edema From ARDS

\begin{tabular}{lcc}
\hline \hline & \multicolumn{2}{c}{ Percentage } \\
\cline { 2 - 3 } & ARDS & Edema \\
\hline Pleural irregularities & 100 & 25 \\
Reduced gliding signs & 100 & 0 \\
Spared areas & 100 & 0 \\
Parenchymal consolidation & 83 & 0 \\
Pleural effusion & 66 & 95 \\
Lung pulse & 50 & 0 \\
\hline
\end{tabular}

reduction of at least $5 \mathrm{~mm} \mathrm{Hg}$ are considered satisfactory. In all patients, there was correlation between the level of efficiency achieved using the 2 methods.

A recent prospective study, including 40 consecutive patients with ARDS/ALI (acute lung injury), demonstrates that lung ultrasound can be used as a reliable tool for assessing PEEP-induced lung recruitment at the bedside. ${ }^{16}$ The authors found a significant correlation between an ultrasound reaeration score and PEEP-induced lung recruitment measured by pressure-volume curves.

The use of CUS in patients with ALI/ARDS has been proposed to assess initial abnormalities of lung morphology and to monitor the effects of therapy ${ }^{1}$

\section{The Role of Ultrasound in Central Venous Catheter Placement and Arterial Catheterization}

For various reasons, patients in ICUs often require central venous catheter placement, for hemodynamic monitoring, administration of drugs, liquids, blood products, or parenteral nutrition. In most cases, the catheters are successfully placed using anatomic findings. In some cases these risky maneuvers can cause complications, some of which are serious, such as PNX, hematoma, and the pricking of a nerve or artery. ${ }^{17}$ To reduce risks and complications, ultrasound has been used over the last few years to locate central veins. ${ }^{18}$

Many authors have shown the effectiveness of ultrasound in this field, ${ }^{19}$ particularly when placing a central venous catheter in an internal jugular vein; fewer maneuvers are needed, compared to approaches based on anatomical findings, and there are fewer complications. ${ }^{20}$

The echo-guided techniques of central venous catheter placement are also recommended for children ${ }^{21}$ and when maneuvers are difficult, such as in obese patients and in patients with short necks. ${ }^{22}$

The radial artery is the preferred site for arterial catheterization; it is a relatively safe procedure, with a low incidence of permanent ischemic complications. A recent systematic review highlights the utility of real-time 2-dimensional ultrasound guidance for radial artery catheterization: compared with the palpation method, the technique was associated with a $71 \%$ improvement in the likelihood of first-attempt success..$^{23}$ The procedure ${ }^{24}$ is easy to learn, particularly if the clinician is already familiar with CUS guidance for central venous catheter placement.

\section{Limitations}

The correct use of CUS requires appropriate training to acquire the necessary knowledge and technical skills. The limitations are those related to the use of ultrasound in other anatomical areas: the skill of the operator plays an 


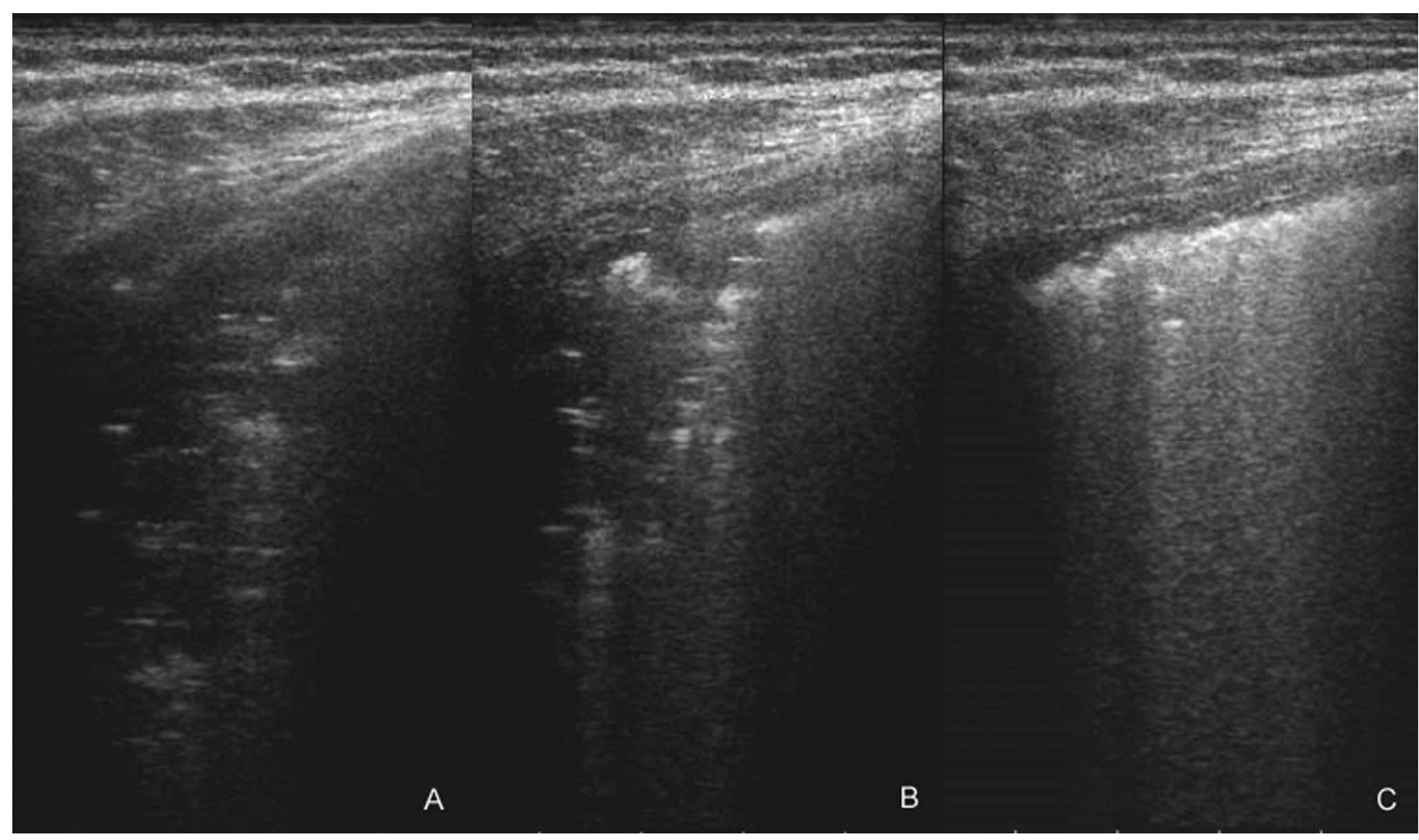

Fig. 13. Recruitment maneuver ultrasound evaluation. A: Consolidated parenchymal area in patient suffering from ARDS. B: The hyperreflectant air aspect reaching the pulmonary consolidation and the progressive reduction of its compactness. C. Recruitment: consolidation is dimensionally reduced and partly replaced by normal air parenchyma, full of "B lines", an expression of interstitial thickening.

important role. Moreover, it is difficult to use this technique on obese patients with thick chest walls ${ }^{1}$; in addition, the technique's efficiency is limited in patients with subcutaneous emphysema. In ICUs, patients often lie in non-optimal positions, which limits the exploration of certain lung areas. Furthermore, the presence of drains and catheters can interfere with the appropriate placement of the transducer.

\section{Summary}

CUS is an accurate method for the diagnosis of PNX, alveolar-interstitial syndromes, parenchymal consolidations, and pleural effusion. The technique holds potential for the evaluation of recruitment maneuvers. It is an easily available, user-friendly, inexpensive medical technique that involves no ionizing radiation. CUS is an interesting medical method that is complementary to bedside CXR and reduces the need to use a CT scan. In the future, CUS will be used increasingly in the ICU.

\section{REFERENCES}

1. Bouhemad B, Zhang M, Lu Q, Rouby JJ. Clinical review: bedside lung ultrasound in critical care practice. Crit Care 2007;11(1):205.
2. KirkpatricK AW, Sirois M, Lupland KB, Liu D, Roman K, Ball CG, et al. Hand-Held thoracic sonography for detection post-traumatic pneumothoraces: the extended focused assessment with sonography for trauma (EFAST). J Trauma 2004;57(2):288-295.

3. Lichtenstein D, Menu Y. A bedside ultrasound sign ruling out pneumothorax in the critically ill: lung sliding. Chest 1995;108(5):13451348.

4. Soldati G, Testa A, Sher S, Pignataro G, La Sala M, Silveri NG. Occult traumatic pneumothorax: accuracy of lung ultrasonography in the emergency department. Chest 2008;133(1):204-211.

5. Lichtenstein D, Mezière G, Biderman P, Gepner A. The "lung point": an ultrasound sign specific to pneumothorax. Intensive Care Med 2000;26(10):1434-1440.

6. Piccoli M, Trambaiolo P, Salustri A, Cerquetani E, Posteraro A, Pastena G, et al. Bedside diagnosis and follow-up of patients with pleural effusion by a hand-carried ultrasound device early after cardiac surgery. Chest 2005;128(5):3413-3420.

7. Soldati G, Copetti R. Ecografia toracica. Torino: CG Edizioni Medico Scientifiche; 2006:31-42.

8. Eibenberger WI Dock, ME Ammann, Dorffner R, Hörmann MF, Grabenwöger F. Quantification of pleural effusion: sonography versus radiography. Radiology 1994;191(3):681-684.

9. Beckh S, Bölcskei PL, Lessnau KD. Real-time chest ultrasonography. a comprehensive review for the pulmonologist. Chest 2002; 122(5):1759-1773.

10. Roch A, Bojan M, Michelet P, Romain F, Bregeon F, Papazian L, et al. Usefulness of ultrasonography in predicting pleural effusions $>500 \mathrm{~mL}$ in patients receiving mechanical ventilation. Chest 2005; 127(1):224-232. 


\section{Chest Ultrasonography IN THE ICU}

11. Lichteinstein D, Mezière G. Relevance of lung ultrasound in the diagnosis of acute respiratory failure: the blue protocol. Chest 2008; 134(1):117-125.

12. Copley SJ, Wells AU, Hawtin KE, Gibson DJ, Hodson JM, Jacques AE, Hansell DM. Lung morphology in the elderly: comparative CT study of subjects over 75 years old versus those under 55 years old. Radiology 2009;251(2):566-573.

13. Copetti R, Soldati R, Copetti P. Chest sonography: a useful tool to differentiate acute cardiogenic pulmonary edema and acute respiratory distress syndrome. Cardiovasc Ultrasound 2008;6:16.

14. Caironi $\mathrm{P}$, Gattinoni L. How to monitor lung recruitment in patients with acute lung injury. Curr Opin Crit Care 2007;13(3):338-343.

15. Gardelli G, Feletti F, Gamberini E, Bonarelli S, Nanni A, Mughetti M. Using sonography to assess lung recruitment in patients with acute respiratory distress syndrome. Emerg Radiol 2009;16(3):219221.

16. Bouhemad B, Brisson H, Le-Guen M, Arbelot C, Lu Q, Rouby JJ. Bedside ultrasound assessment of positive end-expiratory pressureinduced lung recruitment. Am J Respir Crit Care Med 2011;183(3): 341-347.

17. Domino KB, Bowdle TA, Posner KL, Spitellie PH, Lee LA, Cheney FW. Injuries and liability related to central vascular catheters: a closed claims analysis. Anesthesiology 2004;100(6):1411-1418.
18. Skolnick ML. The Role of Sonography in the Placement and Management of Jugular and Subclavian Central Venous Catheters. AJR Am J Roentgenol 1994;163(2):291-295.

19. Miller AH, Roth BA, Mills TJ, Woody JR, Longmoor CE, Foster B. Ultrasound guidance versus the Landmark technique for the placement of central venous catheters in the emergency department. Acad Emerg Med 2002;9(8):800-805.

20. Cavanna L, Civardi G, Vallisa D, Di Nunzio C, Cappucciati L, Bertè $\mathrm{R}$, et al. Ultrasound-guided central venous catheterizationin cancer patients improves the success rate of cannulation and reduces mechanical complications: a prospective observational study of 1,978 consecutive catheterizations. World J Surg Oncol 2010 19;8:91.

21. Pirotte T, Veyckemans F. Ultrasound-guided subclavian vein cannulation in infants and children: a novel approach. Br J Anaesth 2007;98(4):509-514.

22. Troianos CA, Jobes DA, Ellison N. Ultrasound-guided cannulation of the intemal jugular vein: a prospective, randomized study. Anesth Analg 1991;72(6):823-826.

23. Shiloh AL, Savel RH, Paulin LM, Eisen LA. Ultrasound-guided catheterization of the radial artery: a systematic review and metaanalysis of randomized controlled trials. Chest 2011;139(3):524-529.

24. Shiloh AL, Eisen LA. Ultrasound guided arterial catheterization. Intensive Care Med 2010;36(2):214-221. 